\title{
A Review of Organ Dose Calculation Tools for Patients Undergoing Computed Tomography Scans
}

\author{
Choonsik Lee \\ Division of Cancer Epidemiology and Genetics, National Cancer Institute, National Institutes of Health, Rockville, MD, USA
}

\section{Review}

Received April 14, 2021

Revision May 18, 2021

Accepted June 3, 2021

Corresponding author: Choonsik Lee

Division of Cancer Epidemiology and Genetics, National Cancer Institute, National Institutes of Health, 9609 Medical Center Drive, Rockville, MD 20850, USA

E-mail: choonsik.lee@nih.gov

(iD) https://orcid.org/0000-0002-6219-6622

This paper is an invited paper recommended by the Korean Association for Radiation Protection (KARP).

This is an open-access article distributed under the terms of the Creative Commons Attribution License (http://creativecommons.org/licenses/by-nc/4.0), which permits unrestricted use, distribution, and reproduction in any medium, provided the original work is properly cited.

Copyright $\odot 2021$ The Korean Association for Radiation Protection

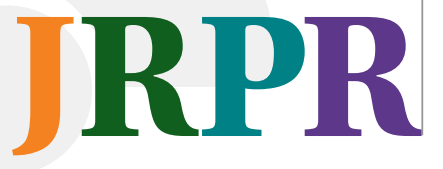

Background: Computed tomography (CT) is one of the crucial diagnostic tools in modern medicine. However, careful monitoring of radiation dose for CT patients is essential since the procedure involves ionizing radiation, a known carcinogen.

Materials and Methods: The most desirable CT dose descriptor for risk analysis is the organ absorbed dose. A variety of CT organ dose calculators currently available were reviewed in this article.

Results and Discussion: Key common elements included in CT dose calculators were discussed and compared, such as computational human phantoms, CT scanner models, organ dose database, effective dose calculation methods, tube current modulation modeling, and user interface platforms.

Conclusion: It is envisioned that more research needs to be conducted to more accurately map CT coverage on computational human phantoms, to automatically segment organs and tissues for patient-specific dose calculations, and to accurately estimate radiation dose in the cone beam computed tomography process during image-guided radiation therapy.

Keywords: Computed Tomography, Organ Dose, Computational Human Phantoms, Monte Carlo

\section{Introduction}

Computed tomography (CT) is one of the crucial diagnostic tools in modern medicine. However, careful monitoring of radiation dose for CT patients is essential since the procedure involves ionizing radiation, a known carcinogen. There is direct evidence of adverse late effects reported from multiple epidemiological studies of pediatric CT patients [1-3]. Pediatric patients, in particular, have garnered increased attention since they are known to be more vulnerable to ionizing radiation due to their more active cell development and longer life expectancy compared to adults [4]. To make an informed decision on the use of $\mathrm{CT}$, it is critical to define relevant dose metrics and to accurately estimate them.

Several dose quantities concerning CT, called CT dose descriptors, are available from CT scanners [5]: weighted CT dose index $\left(\mathrm{CTDI}_{\mathrm{w}}\right)$, volumetric CT dose index (CTDI $\left.{ }_{\mathrm{vol}}\right)$, and dose-length product (DLP). However, these dose descriptors are basically derived from $\mathrm{CTDI}_{100}$, the radiation dose delivered to a $100 \mathrm{~mm}$ long ion chamber plugged in an acrylic cylinder phantom. However, since the cylindrical shape and homogeneity of the CTDI phantoms are different from those of the human anatomy, these CT dose de- 
scriptors cannot be considered as doses delivered to a patient. There is a "derived" dose descriptor, called effective dose, which is more relevant to patient doses. Effective dose is defined as the sum of the equivalent doses delivered to major radiosensitive organs weighted by tissue weighting factors [5]. To properly estimate the effective dose, absorbed doses to multiple radiosensitive organs are required as discussed later in this article. However, DLP-to-effective dose conversion factors, called $\mathrm{k}$-factor [6], can be conveniently used to derive effective doses from DLP, which is readily available from CT scanners. Nonetheless, the effective dose is defined as ageand gender-averaged quantity, so cannot be used to estimate the risk of late effects from CT scans. The most desirable CT dose descriptor for risk analysis is organ absorbed dose [7].

There are two different approaches to estimating organ doses for CT patients. First, organ doses can be measured by using a surrogate patient anatomy model, called a physical anthropomorphic phantom. Different types of radiation dosimeters are embedded into the physical phantoms before CT scans and processed to read dose after scans. The procedure is often laborious and expensive. Second, organ doses can be calculated through the computer simulations of CT scans where CT scanner simulation models and the anatomical surrogate of a patient, called computational human phantoms, are involved. The simulation approach is more flexible and cost-effective than measurement so many researchers have developed different computational approaches, some of which then led to the development of user-friendly dose calculation tools for CT patients. To date, various CT dose calculation tools have been introduced to the radiology community, but little information is available concerning the comparison of their key elements.

In the current review article, a variety of CT dose calculation tools currently available will be reviewed. Common technical elements in CT dose calculators will be described and compared. Ongoing and future research on CT patients' dosimetry will be discussed.

\section{Elements of CT Dose Calculators}

The summary of the elements in different CT dose calculators currently available is tabulated in Table 1 [8-15]. Several

Table 1. List of the CT Organ Dose Calculation Tools Currently Available as of 2021

\begin{tabular}{|c|c|c|c|c|c|c|c|c|}
\hline \multirow{2}{*}{$\begin{array}{l}\text { Program } \\
\text { name }\end{array}$} & \multicolumn{4}{|c|}{ Computational human phantoms } & \multirow{2}{*}{$\begin{array}{l}\text { Tube current } \\
\text { modulation }\end{array}$} & \multirow{2}{*}{$\begin{array}{l}\text { User interface } \\
\text { platforms }\end{array}$} & \multirow[b]{2}{*}{ Availability } & \multirow[b]{2}{*}{ References } \\
\hline & $\begin{array}{c}\text { Reference size } \\
\text { pediatric }\end{array}$ & $\begin{array}{l}\text { Reference size } \\
\text { adult }\end{array}$ & Size-dependent & $\begin{array}{l}\text { Pregnant } \\
\text { women }\end{array}$ & & & & \\
\hline CT-Expo & $\begin{array}{l}\text { GSFa) voxel }^{\text {a }} \\
\quad(n=2)\end{array}$ & GSF stylized $(n=2)$ & - & - & - & $\begin{array}{l}\text { Standalone } \\
\text { Excel }\end{array}$ & Commercial & {$[11]$} \\
\hline DoseWatch & \multicolumn{4}{|c|}{ XCAT $^{\mathrm{b})}$ hybrid $(\mathrm{n}=150)$} & Retrospective & PACS $^{\mathrm{c})}$-embedded & Commercial & {$[12]$} \\
\hline ImPACT & - & $\begin{array}{l}\text { ORNL d) stylized } \\
(n=2)\end{array}$ & - & - & - & $\begin{array}{l}\text { Standalone } \\
\text { Excel }\end{array}$ & Commercial & [8] \\
\hline ImpactDose & $\begin{array}{l}\text { ORNL stylized } \\
(n=10)\end{array}$ & $\begin{array}{l}\text { ORNL stylized } \\
(n=2), \operatorname{ICRP}(n=2)\end{array}$ & - & - & Prospective & $\begin{array}{l}\text { Standalone } \\
\text { Windows }\end{array}$ & Commercial & - \\
\hline $\mathrm{NClCT}$ & $\begin{array}{l}\text { (CRPe) voxel } \\
\quad(n=10)\end{array}$ & ICRP voxel $(n=2)$ & $\begin{array}{l}\mathrm{NCl}^{\dagger} \text { hybrid pediatric } \\
\text { and adult }(\mathrm{n}=351)\end{array}$ & $\begin{array}{l}\text { UFg) hybrid }^{\text {hal }} \\
\quad(n=8)\end{array}$ & $\begin{array}{c}\text { Retrospective/ } \\
\text { prospective }\end{array}$ & $\begin{array}{l}\text { Standalone } \\
\text { Windows, Mac, } \\
\text { Linux }\end{array}$ & Free & {$[13]$} \\
\hline $\begin{array}{l}\text { VirtualDose } \\
\text { CT }\end{array}$ & $\begin{array}{l}\text { RPI }^{\text {h) }} \text { hybrid } \\
(n=10)\end{array}$ & RPI hybrid $(n=2)$ & $\begin{array}{l}\text { RPI hybrid adult } \\
\qquad(n=10)\end{array}$ & $\begin{array}{l}\text { RPI hybrid } \\
\qquad(n=3)\end{array}$ & Retrospective & Web application & Commercial & {$[14],[15]$} \\
\hline Radimetrics & $\begin{array}{l}\text { ORNL stylized } \\
(n=10)\end{array}$ & ORNL stylized $(n=2)$ & - & $\begin{array}{l}\text { ORNL stylized } \\
(n=3)\end{array}$ & Retrospective & PACS-embedded & Commercial & {$[10]$} \\
\hline WinDose & - & GSF stylized $(n=2)$ & - & - & - & $\begin{array}{l}\text { Standalone } \\
\text { Windows }\end{array}$ & Commercial & {$[9]$} \\
\hline
\end{tabular}

The programs are in an alphabetical order.

CT, computed tomography; ICRP, International Commission on Radiological Protection; NCICT, National Cancer Institute dosimetry system for computed tomography.

a) National Research Center for Environment and Health (current Helmholtz Zentrum Munchen).

b) eXtended CArdiac-Torso. From the website, it is not disclosed how many phantoms are included in different phantom categories.

c)Picture Archiving and Communication System.

d) Oak Ridge National Laboratory.

e)International Commission on Radiological Protection.

i) National Cancer Institute.

g) University of Florida.

h) Rensselaer Polytechnic Institute. 
web applications simply providing effective doses or wholebody fetal doses are also available. However, the scope of the review was limited to CT dose calculation tools that calculate organ-level doses. CT dose calculators are commonly composed of multiple elements: computational human phantoms, CT scanner models, organ dose database, the algorithms of effective dose calculation, and tube current modulation modeling, which are housed in different types of platforms.

\section{Computational Human Phantoms}

Patient anatomy is one of the most critical elements of CT dose calculators. The most accurate anatomy model in CT dose calculations would be the anatomy of a patient, which possibly can be reconstructed from CT images. However, there are several challenges in this option including automatic segmentation of organs of interest, which will be discussed later. The next best anatomy models are computational human phantoms, one of the rapidly developing areas in medical physics over the past decades [16]. The computational phantoms are developed by contouring major organs of interest from CT or magnetic resonance (MR) images of patients and are adjusted to match the dimensions of the body and internal organs to those of the reference (or standard) human models established by a comprehensive literature search such as the International Commission on Radiological Protection (ICRP) Publication 89 [17]. As shown in Table 1, different libraries of computational human phantoms are implemented into CT dose calculators: age-dependent reference size pediatric and adult phantoms, body size-dependent phantoms, and pregnant women phantoms with fetal models. Depending on the availability of patient information, CT dosimetrists can choose different sets of phantoms for organ dose calculations.

When the only information available for a patient is age and gender, one can choose age and gender-dependent phantoms with reference body sizes. When this option is adopted, the impact of body size on organ dose would be ignored. Three categories of age-dependent phantoms have been introduced in the past decades. The first series of age-dependent phantoms were created using mathematical equations with Boolean operations, which are called mathematical or stylized phantoms [18]. ImPACT [8] and WinDose [9] employed adult male and female stylized phantoms developed at the Oak Ridge National Laboratory (ORNL). ImPACT program also provides a conversion table to scale the adult dose to that of pediatric patients. ImpactDose [19] and Radimetrics [10] adopted a series of pediatric and adult stylized phantoms. However, the mathematical equations and Boolean operations used to describe the anatomical structure of the stylized phantoms are limited to model the complicated structures of the human anatomy. To address the limitation, a new generation of phantoms based on tomographic images, called voxel phantoms [20, 21], were developed from CT images of pediatric and adult patients. CT-Expo [11] was the first CT dose calculator which included pediatric voxel phantoms. However, the program displays stylized-looking pediatric phantoms, whereas the software manual shows that the voxel phantoms, Baby and Child [22], were used for dose calculations. A more complete series of pediatric voxel phantoms were developed by the University of Florida research team [21] but were not implemented into CT dose calculators. Following voxel phantoms, a new phantom construction method using polygon mesh or non-uniform rational B-spline (NURBS) was used to develop a library of pediatric and adult phantoms, called hybrid phantoms (meaning combining the advantages of stylized and voxel phantoms) [23-25]. Different series of age-dependent hybrid phantoms were implemented in the DoseWatch [12], National Cancer Institute dosimetry system for CT (NCICT) [13], and VirtualDose CT [14]. Fig. 1 shows the frontal views of the stylized adult male phantom in CTExpo 2.1, the ICRP adult male voxel phantom in ImpactDose 2.2, and the National Cancer Institute adult male hybrid phantom in NCICT 2.0.

The impact of the variation of body sizes even at the same age on organ dose in CT dosimetry has been largely investigated and highlighted the importance of implementing body size-dependent phantoms into CT dose calculators. The modeling flexibility of hybrid phantoms made it possible to modify reference size phantoms to create body size-dependent phantoms by adjusting the shape and dimension of phantoms composed of a polygon mesh and NURBS surfaces. DoseWatch [12] features a library of body-size-dependent adult XCAT phantoms [25]. VirtualDose CT also has a series of ten body size-dependent adult male and female phantoms [26]. NCICT 2.0 [27] implements a library of 351 pediatric and adult hybrid phantoms [28] with a range of height and weight.

In addition to the anatomical differences caused by age and body size, another dramatic change in human anatomy happens during pregnancy. Due to its high radiosensitivity and risk of adverse late effects, the fetus is of particular interest in CT dosimetry. Following the early stylized pregnant 

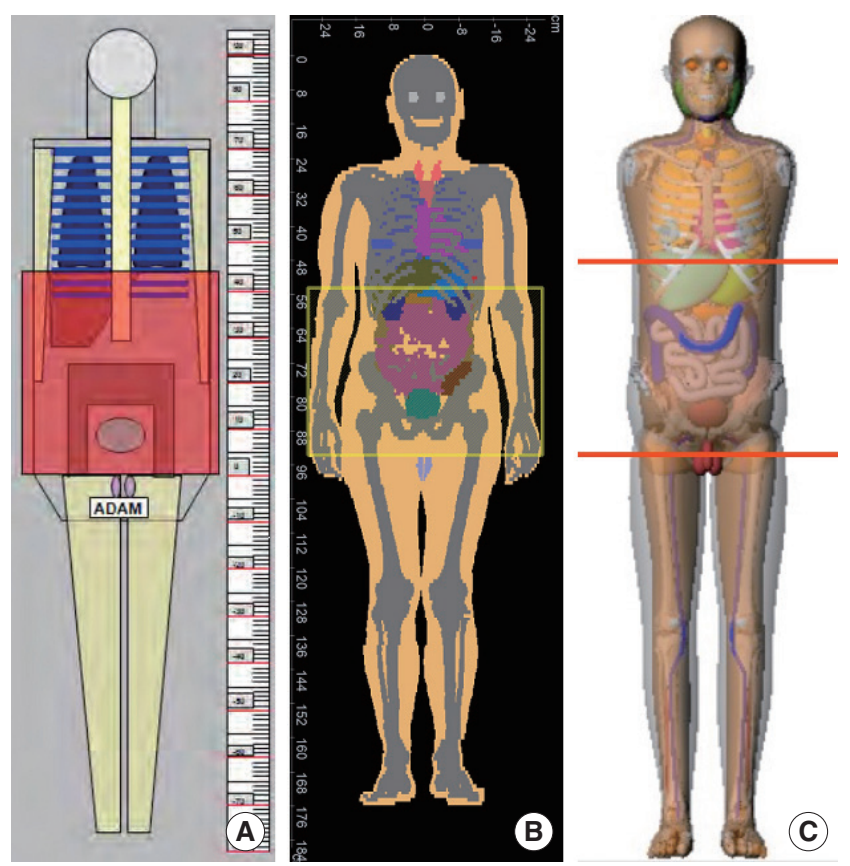

Fig. 1. Frontal views of the stylized adult male phantom, the International Commission on Radiological Protection (ICRP) adult male voxel phantom, and the National Cancer Institute ( $\mathrm{NCl}$ ) hybrid adult male phantom, that are built in (A) CT-Expo 2.1, (B) ImpactDose 2.2, and (C) NCICT 2.0, respectively.

women phantoms [29] which are implemented in Radimetrics [10], the voxel [30] and hybrid [30,31] pregnant women phantoms have evolved taking advantage of new phantom technologies. DoseWatch implements a series of XCAT hybrid pregnant phantoms for CT dose calculations. A library of eight pregnant women phantoms from the University of Florida research team is adopted in NCICT 3.0 [32]. VirtualDose CT calculates the dose to the fetus using three RPI hybrid pregnant women phantoms [33].

\section{CT Scanner Models}

Technical parameters in CT scanners such as tube potential, bowtie filter, collimation, fan beam angle, etc., make differences in the characteristics of X-ray beams, which result in differences in radiation dose delivered to patients. To accurately implement scanner-specific beam characteristics into organ dose calculations, detailed technical parameters should be used in the Monte Carlo radiation transport simulations of CT scanners. The modeling process of CT X-rays has been published by several researchers [34-37] but usually follows the steps below. The material composition and dimension of the head and body filters can be obtained from manufacturers. Different X-ray spectra corresponding to the filters are usually generated using an X-ray spectrum generation program such as SPECT78 [38]. Bowtie filters can be explicitly modeled using the detailed composition and dimensions obtained from manufacturers. However, to increase the efficiency of Monte Carlo simulations, weighting factors estimated from a lateral dose profile in a pencil ion chamber are applied to angular sampling within a fan beam angle [36] . The X-ray source is generated at a location defined by a focalspot-to-axis distance, which is also obtained from manufacturers. Once CT X-ray source modeling is completed, the resulting CTDI values at the center and periphery of the $16 \mathrm{~cm}$ and $32 \mathrm{~cm}$ CTDI phantoms from the simulations are validated by comparing with measurements at different tube potentials and filtrations.

However, technical specifications of CT scanners are only available from scanner manufacturers and are often proprietary. ImPACT [8] contains a library of organ dose databases generated by modeling several CT scanners based on manufacturer-provided scanner-specific technical parameters. The software also has a "matching" feature to extend the simulated CT scanner models to new scanner models which were not included in the Monte Carlo simulations. However, Monte Carlo simulations of different scanners are time-consuming, and the approach is not flexible to cover a variety of CT scanners that are currently available at clinics and will be introduced.

To make CT dose calculators more flexible, it was suggested that the dependency of organ doses on CT scanners' characteristics becomes minimal when organ doses calculated from a scanner model are normalized to CTDI ${ }_{\mathrm{vol}}$ measured from the given scanner. That is, although the organ doses from scanner A are different from those from scanner B, the organ doses per CTDI ${ }_{\text {vol }}$ of scanner A become very close to those per CTDI ${ }_{\text {vol }}$ of scanner B within coefficient of variation, $5 \%$ [39]. When organ doses for a new CT scanner are sought, the

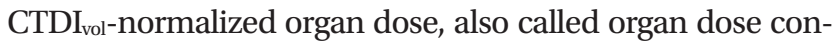
version coefficients, can be multiplied by $\mathrm{CTDI}_{\mathrm{vol}}$ from the given scanner. The scanner-independent organ dose algorithm has been widely adopted in most CT dose calculators available to date [39].

\section{Organ Dose Database}

All CT dose calculators reviewed in the current article employ a precalculated organ dose database to perform rapid dose calculations. Organs and tissues to be included in the organ dose database are selected based on their radiosensi- 
tivity following the list of organs to which ICRP assigns tissue weighting factors. According to ICRP Publication 103 [40], the essential organs and tissues such as the red bone marrow, colon, lungs, stomach, and breast with the tissue weighting factor of 0.12 are always included in the list of organs in CT dose calculators. The primary organ group is followed by the gonads (testes for males and ovaries for females) with their tissue weighting factor, 0.08 . The organs with a tissue weighting factor of 0.04 are then included such as the bladder wall, esophagus, liver, and thyroid. The remaining organs include the bone surface (also known as the endosteum), brain, salivary glands (parotid, sublingual, and submandibular), and skin, of which tissue weighting factor is 0.01 . The ICRP groups several organs as "remainder tissues" and assigns the tissue weighting factor of 0.12: adrenals, extrathoracic region, gallbladder, heart, kidneys, lymphatic nodes, muscle, oral mucosa, pancreas, prostate for males, small intestine, spleen, thymus, uterus/cervix for females. The full list of the radiosensitive organs and tissues is not usually available in the stylized phantoms and not always available even in the modern voxel and hybrid phantoms so that some CT dose calculators use surrogate organs and tissues. Use of the surrogate organs in CT dose calculations may result in dosimetric errors [41].

CT dose calculators adopt an approach to approximate helical scans by the summation of doses from multiple axial slices included in the given scan range of interest. To create an organ dose database, a large amount of Monte Carlo calculations is required. For a given phantom, dose calculations for a series of axial slices must be conducted from the top of the head to the bottom of the feet of the phantom with a certain slice increment. For example, when an adult phantom $175 \mathrm{~cm}$ tall is adopted and the slice thickness $1 \mathrm{~cm}$ is desired, a total of 175 Monte Carlo calculations must be conducted. The number of calculations increases by the combination of the X-ray spectrum and the number of phantoms. As mentioned in the previous section, the resulting organ doses (mGy) are normalized by CTDI ${ }_{\mathrm{vol}}$ (mGy) to establish CTDI vol $^{-}$ to-organ dose conversion coefficients (mGy/mGy or unitless), which are independent of CT scanner models.

\section{Effective Dose Calculation Methods}

As mentioned above, the effective dose is widely used as a derived CT dose descriptor in CT dosimetry. However, it is often unclear to the users what the quantity means and how it is derived [42]. There are two approaches to estimating ef- fective doses for CT patients. First, the effective dose can be calculated by using DLP to effective dose conversion factors, called $\mathrm{k}$-factor [6], which is adopted by many CT dose reporting systems. The effective dose for a chest CT scan for an adult patient, for example, can be estimated by multiplying the conversion factors defined for chest scans and adults by the DLP value displayed on the CT dose page. However, the conversion factors are often blindly used without acknowledging how the values are derived and what uncertainty factors are involved. It is critical to consider several factors affecting $\mathrm{k}$ factors.

$\mathrm{k}$-factors change depending on which computational human phantoms are used. The $k$-factors reported by the American Association of Physicists in Medicine (AAPM) [6] were calculated using the ORNL stylized phantoms, of which anatomical structures are not realistic compared to human anatomy as mentioned above. The organs included in the scan range of the chest scan in the ORNL stylized phantoms may not be the same as those covered in the chest scan. Since the torso of the stylized phantoms is reported to be narrower in anteroposterior direction compared to the human body, organ doses calculated from stylized phantoms tend to overestimate the doses estimated by using realistic voxel or hybrid phantoms. This implies that the effective dose derived from the stylized phantom-based $k$-factors may be greater than that from voxel or hybrid phantoms. $\mathrm{k}$-factors also depend on which set of tissue weighting factors is used. The ICRP has published three sets of tissue weighting factors to date in ICRP Publication 26 [43], ICRP Publication 60 [44], and ICRP Publication 103 [40]. All CT dose calculators but ImPACT program estimate and display effective dose based on the latest tissue weighting factors from ICRP Publication 103 as well as effective dose based on the tissue weighting factors from ICRP Publication 60. Different sets of tissue weighting factors change the resulting effective dose by up to $40 \%$ between ICRP Publications 60 and 103 [16]. Lastly, it is suggested to account for body size in effective dose [45, 46]. It is found that reference size phantoms-based effective dose may underestimate effective dose received by underweight patients and overestimate effective dose received by overweight patients when not accounting for body size in the calculations of organ doses and corresponding effective doses [46].

The second method to derive effective dose for CT patients directly uses organ doses calculated for a given patient not relying on $\mathrm{k}$-factors. All CT dose calculators reviewed in this article provide both $\mathrm{k}$-factor-based and organ dose-based ef- 
fective doses. To correctly follow the definition of effective dose by ICRP, gender-specific organ doses must be averaged before summing equivalent doses weighted by tissue weighting factors [40]. This gender neutralization was reflected in kfactors, which are not specified by gender. However, when effective dose is calculated from organ doses from a male or female phantom, that is a pseudo-effective dose different from the ICRP definition.

\section{Tube Current Modulation Modeling}

One of the dose reduction techniques adopted in modern CT scanners is tube current modulation (TCM), which is one branch under automatic exposure control techniques. The basic idea of TCM is to modulate the electric current of the $\mathrm{x}$ ray tube depending on the signal-to-noise ratio which varies at different angles within a single slice due to the oval crosssectional shape of the human body (e.g., the thickness difference between anteroposterior and lateral geometries) and at different longitudinal locations due to the difference of tissue density (e.g., the density around the lungs is smaller than that around the pelvic area). The purpose of this technique is to maintain a consistent image signal-to-noise ratio in the CT image across a patient's anatomy with varying degrees of attenuation to reduce radiation dose to patients while maintaining image quality.

There are two approaches to implement modulated tube currents into organ dose calculations. First, modulated tube current can be extracted from the Digital Imaging and Communications in Medicine (DICOM) header or Radiation Dose Structured Report (RDSR) after CT scans [46], which is called a retrospective approach. Because the algorithm of TCM techniques varies by CT scanner manufacturers and is proprietary, the major advantage of this post-scan method is that the actual modulated current profiles can be used for organ dose calculations. Several CT dose calculators use this algorithm to account for TCM in organ dose calculations [10, 12-14] as shown in Table 1.

However, in some cases, dosimetrists may need to predict organ doses before CT scans are conducted or calculate generic organ doses not specific to a certain CT scanner model, which is called a prospective approach. Although the algorithm of TCM varies across different manufacturers, the fundamental goal is to reduce the dose while minimizing image noise by modulating the current of the X-ray tube depending on the signal-to-noise ratio. There are generic algorithms reported to derive TCM profiles from computational human phantoms [47-50]. The amount of attenuation is calculated following the X-ray beam path starting from the source through the phantom's anatomy to the detector within the fan beam angle at a single source rotational angle, which is repeated from $0^{\circ}$ to $360^{\circ}$ following a rotating CT source. Angular modulation profiles can be averaged to create a modulation profile for a single slice, which is repeated from the top of the head to the bottom of the feet in a phantom. This algorithm was adopted in DoseWatch, ImpactDose, and NCICT 2.0. Some studies report the organ dose difference between TCM and fixed tube current $[13,47,49,51]$.

\section{User Interface Platforms}

CT dose calculators are available on different platforms and each platform has pros and cons. First, some programs are operating as stand-alone programs. CT-Expo and ImPACT are based on the Microsoft Excel program using Visual Basic for Applications (VBA) technology. The size of the program is small and the programs will not need to be installed. One drawback of the platform is a compatibility issue occurring whenever Microsoft Excel is upgraded. Other programs such as NCICT, ImpactDose, and WinDose are operating on Windows operating system as a stand-alone program without relying on additional programs such as Microsoft Excel. NCICT is also running on Mac and Linux. Second, VirtualDose CT is running on a web-based server. The users should upload their CT parameters or images to the web application to calculate organ doses. This platform is more efficient for update and maintenance compared to the stand-alone programs. However, the operation requires an internet connection and patient data transfer may be an issue for some clinical settings although they are anonymized. Third, other programs such as DoseWatch and Radimetrics are embedded in the Picture Archiving and Communication System (PACS). The patient data are readily transferred to the dose calculation modules within the PACS. The results are directly used for dose monitoring purposes and will help to make informed decisions.

\section{Ongoing and Future Research}

There are three major areas in CT organ dosimetry that are under active development and need more work in the future. First, although the organ dose calculation methods and tools are well developed as mentioned above, it is still challenging to map the patient's scan range onto the computational hu- 
man phantoms implemented in CT dose calculators. One method to do this is to use the anatomical locations defined in general scan protocols. For example, chest scan protocols usually start from the top point of the clavicle to the middle point of the liver. One can use the same protocol ignoring the actual scan ranges conducted on a patient whenever organ doses for chest CT patients are sought. This approach is generally used for a large-scale cohort of patients. However, it is reported that general scan protocols are not always strictly followed [46] even in a clinical trial where protocols are carefully designed and provided to participating hospitals. To avoid dose errors caused by a disagreement between a patient and phantom, automatic mapping methods have been investigated [52] that compare patient CT images with predefined anatomical landmarks in computational human phantoms to map the locations of scan start and end of a patient on those of phantoms. Machine learning could be used for mapping organs from CT images to computational human phantoms [53].

Second, although reference computational human phantoms and body size-matched phantoms are used to more accurately simulate a patient's anatomy, there are still discrepancies between a phantom and a patient. As briefly mentioned above, the most accurate anatomical model would be the one constructed from the CT images of a given patient. To utilize CT images for organ dose calculations, however, the organs of interest need to be rapidly segmented. Recent studies used deep learning-based automatic segmentation methods to contour major radiosensitive organs from CT images [15, 54, 55]. Some organs such as the intestines are still challenging to segment due to their complicated and variable shapes so the pre-contoured organ models from computational phantoms are used as alternatives. The segmented patient models are coupled with fast radiation transport methods often based on graphical processing unit (GPU)-based Monte Carlo tools [56].

Lastly, as radiation dose delivered to patients from imaging procedures involved in the image-guided radiation therapy (IGRT) increases due to the advance of modern imaging techniques, more attention has been given to the needs of the accurate estimation of imaging dose and its implementation into treatment planning. The imaging dose from a single $\mathrm{kV}$ cone-beam computed tomography (CBCT) acquisition may be low compared to the therapy dose. However, the normal tissue dose can be substantially increased by repeated daily imaging procedures throughout many treatment frac- tions. Several researchers report algorithms and doses calculated for CBCT [57, 58], but no user-friendly rapid dose calculators are available yet.

\section{Summary}

A variety of CT organ dose calculators currently available were reviewed in this article. Key common elements included in CT dose calculators were discussed and compared such as computational human phantoms, CT scanner models, organ dose database, effective dose calculation methods, tube current modulation modeling, and user interface platforms. It is envisioned that more research needs to be conducted to more accurately map CT coverage on computational human phantoms, to automatically segment organs and tissues for patient-specific dose calculations, and to accurately estimate radiation dose in the CBCT process during IGRT.

\section{Conflict of Interest}

No potential conflict of interest relevant to this article was reported.

\section{Acknowledgements}

This research was funded by the intramural research program of the National Institutes of Health, National Cancer Institute, Division of Cancer Epidemiology and Genetics.

\section{References}

1. Pearce MS, Salotti JA, Little MP, McHugh K, Lee C, Kim KP, et al. Radiation exposure from CT scans in childhood and subsequent risk of leukaemia and brain tumours: a retrospective cohort study. Lancet. 2012;380:499-505.

2. Mathews JD, Forsythe AV, Brady Z, Butler MW, Goergen SK, Byrnes GB, et al. Cancer risk in 680,000 people exposed to computed tomography scans in childhood or adolescence: data linkage study of 11 million Australians. BMJ. 2013;346:f2360.

3. Meulepas JM, Ronckers CM, Smets AM, Nievelstein RJ, Gradowska P, Lee C, et al. Response to Wollschläger, Blettner, and Pokora. J Natl Cancer Inst. 2019;111:1002-1003.

4. National Research Council. Health risks from exposure to low levels of ionizing radiation (BEIR VII Phase 2). Washington, DC: National Academies Press; 2006.

5. McNitt-Gray MF. AAPM/RSNA Physics Tutorial for Residents: topics in CT: radiation dose in CT. Radiographics. 2002;22:1541- 
1553.

6. McCollough CH, Cody DD, Edyvean S, Geise R, Gould B, Keat N, et al. The measurement, reporting, and management of radiation dose in CT. Rep AAPM Task Group. 2008;23:1-28.

7. Simon SL, Kleinerman RA, Ron E, Bouville A. Uses of dosimetry in radiation epidemiology. Radiat Res. 2006;166(1 Pt 2):125-127.

8. ImPACT. ImPACT CT patient dosimetry calculator. London, UK: ImPACT; 2011.

9. Kalender WA, Schmidt B, Zankl M, Schmidt M. A PC program for estimating organ dose and effective dose values in computed tomography. Eur Radiol. 1999;9:555-562.

10. Bayer HealthCare LLC. Radiation dose management [Internet]. Whippany, NJ: Bayer HealthCare LLC; c2021 [cited 2021 Jun 8]. Available from: https://www.radiologysolutions.bayer.com/ products/dosing-software/radiation-dose-management.

11. Stamm G, Nagel HD. CT-Expo: ein neuartiges Programm zur Dosisevaluierung in der CT [CT-expo: a novel program for dose evaluation in CT]. Rofo. 2002;174:1570-1576.

12. GE Healthcare. DoseWatch: optimized radiation dose management [Internet]. Waukesha, WI: GE Healthcare; c2021 [cited 2021 Jun 8]. Available from: https://www.gehealthcare.com/products/ dose-management/dosewatch-dose-monitoring-software-gehealthcare.

13. Lee C, Kim KP, Bolch WE, Moroz BE, Folio LR. NCICT: a computational solution to estimate organ doses for pediatric and adult patients undergoing CT scans. J Radiol Prot. 2015;35:891909.

14. Ding A, Gao Y, Liu H, Caracappa PF, Long DJ, Bolch WE, et al. VirtualDose: a software for reporting organ doses from CT for adult and pediatric patients. Phys Med Biol. 2015;60:5601-5625.

15. Peng Z, Fang X, Yan P, Shan H, Liu T, Pei X, et al. A method of rapid quantification of patient-specific organ doses for CT using deep-learning-based multi-organ segmentation and GPU-accelerated Monte Carlo dose computing. Med Phys. 2020;47:25262536.

16. Xu XG. An exponential growth of computational phantom research in radiation protection, imaging, and radiotherapy: a review of the fifty-year history. Phys Med Biol. 2014;59:R233-302.

17. Valentin J. Basic anatomical and physiological data for use in radiological protection: reference values: ICRP Publication 89. Ann ICRP. 2002;32:1-277.

18. Cristy M. Mathematical phantoms representing children of various ages for use in estimates of internal dose. Oak Ridge, TN: Oak Ridge National Laboratory; 1980.

19. CT Imaging GmbH. ImpactDose v2.2. Bavaria, Germany: CT Imaging $\mathrm{GmbH} ; 2015$.

20. Petoussi-Henss N, Zanki M, Fill U, Regulla D. The GSF family of voxel phantoms. Phys Med Biol. 2002;47:89-106.

21. Lee C, Lee C, Williams JL, Bolch WE. Whole-body voxel phantoms of paediatric patients: UF Series B. Phys Med Biol. 2006;51:
4649-4661.

22. Veit R, Zankl M, Petoussi N, Mannweiler E, Williams G, Drexler G. Tomographic anthropomorphic models, Part I: Construction technique and description of models of an 8 week old baby and a 7 year old child (No. GSF-3-89). Neuherberg, Germany: Gesellschaft fuer Strahlen- und Umweltforschung; 1989.

23. Bolch WE, Eckerman KF, Endo A, Hunt JG, Jokisch DW, Kim $\mathrm{CH}$, et al. ICRP Publication 143: paediatric reference computational phantoms. Ann ICRP. 2020;49:5-297.

24. Lee C, Lodwick D, Hurtado J, Pafundi D, Williams JL, Bolch WE. The UF family of reference hybrid phantoms for computational radiation dosimetry. Phys Med Biol. 2010;55:339-363.

25. Norris H, Zhang Y, Bond J, Sturgeon GM, Minhas A, Tward DJ, et al. A set of $4 \mathrm{D}$ pediatric XCAT reference phantoms for multimodality research. Med Phys. 2014;41:033701.

26. Ding A, Mille MM, Liu T, Caracappa PF, Xu XG. Extension of RPI-adult male and female computational phantoms to obese patients and a Monte Carlo study of the effect on CT imaging dose. Phys Med Biol. 2012;57:2441-2459.

27. Lee C, Liu J, Yao J, Summers R, Folio LR. NCICTX: an organ dose calculator for CT patients with different body sizes. Med Phys. 2018;45(6 Suppl):E690-E690.

28. Geyer AM, O'Reilly S, Lee C, Long DJ, Bolch WE. The UF/NCI family of hybrid computational phantoms representing the current US population of male and female children, adolescents, and adults: application to CT dosimetry. Phys Med Biol. 2014; 59:5225-5242.

29. Stabin MG, Watson EE, Cristy M, Ryman JC, Eckerman KF, Davis $\mathrm{JL}$, et al. Mathematical models and specific absorbed fractions of photon energy in the nonpregnant adult female and at the end of each trimester of pregnancy (No. ORNL/TM-12907). Oak Ridge, TN: Oak Ridge National Laboratory; 1995.

30. Shi C, Xu XG. Development of a 30-week-pregnant female tomographic model from computed tomography (CT) images for Monte Carlo organ dose calculations. Med Phys. 2004;31:24912497.

31. Maynard MR, Long NS, Moawad NS, Shifrin RY, Geyer AM, Fong $\mathrm{G}$, et al. The UF Family of hybrid phantoms of the pregnant female for computational radiation dosimetry. Phys Med Biol. 2014;59: 4325-4343.

32. Lee C, Folio LS. An organ dose calculation tool for fetus at various ages undergoing computed tomography. Proceedings of the Radiological Society of North America (RSNA) $105^{\text {th }}$ Scientific Assembly and Annual Meeting; 2019 Dec 1-6; Chicago, IL.

33. Gu J, Xu XG, Caracappa PF, Liu B. Fetal doses to pregnant patients from CT with tube current modulation calculated using Monte Carlo simulations and realistic phantoms. Radiat Prot Dosimetry. 2013;155:64-72.

34. Lee C, Kim KP, Long DJ, Fisher R, Tien C, Simon SL, et al. Organ doses for reference adult male and female undergoing computed 
tomography estimated by Monte Carlo simulations. Med Phys. 2011;38:1196-1206.

35. Jarry G, DeMarco JJ, Beifuss U, Cagnon CH, McNitt-Gray MF. A Monte Carlo-based method to estimate radiation dose from spiral CT: from phantom testing to patient-specific models. Phys Med Biol. 2003;48:2645-2663.

36. Staton RJ, Lee C, Lee C, Williams MD, Hintenlang DE, Arreola $\mathrm{MM}$, et al. Organ and effective doses in newborn patients during helical multislice computed tomography examination. Phys Med Biol. 2006;51:5151-5166.

37. Gu J, Bednarz B, Caracappa PF, Xu XG. The development, validation and application of a multi-detector CT (MDCT) scanner model for assessing organ doses to the pregnant patient and the fetus using Monte Carlo simulations. Phys Med Biol. 2009;54: 2699-2717.

38. Institute of Physics and Engineering in Medicine. Catalogue of diagnostic x-ray spectra and other data. York, UK: Institute of Physics and Engineering in Medicine; 1997.

39. Turner AC, Zankl M, DeMarco JJ, Cagnon CH, Zhang D, Angel E, et al. The feasibility of a scanner-independent technique to estimate organ dose from MDCT scans: using CTDI $_{\mathrm{vol}}$ to account for differences between scanners. Med Phys. 2010;37:1816-1825.

40. The 2007 Recommendations of the International Commission on Radiological Protection: ICRP publication 103. Ann ICRP. 2007;37:1-332.

41. Lee C, Morton LM, Berrington de González A. A novel method to estimate lymphocyte dose and application to pediatric and young adult CT patients in the United Kingdom. Radiat Prot Dosimetry. 2018;178:116-121.

42. Lee C. How to estimate effective dose for CT patients. Eur Radiol. 2020;30:1825-1827.

43. Recommendations of the International Commission on Radiological Protection: ICRP Publication 26. Ann ICRP. 1977;1:1-80.

44. 1990 Recommendations of the International Commission on Radiological Protection. Ann ICRP. 1991;21:1-201.

45. Romanyukha A, Folio LR, Lamart S, Simon SL, Lee C. Body sizespecific effective dose conversion coefficients for CT scans. Radiat Prot Dosimetry. 2016;172:428-437.

46. Lee C, Flynn MJ, Judy PF, Cody DD, Bolch WE, Kruger RL. Body size-specific organ and effective doses of chest CT screening examinations of the National Lung Screening Trial. Am J Roentgenol. 2017;208:1082-1088.
47. Li X, Segars WP, Samei E. The impact on CT dose of the variability in tube current modulation technology: a theoretical investigation. Phys Med Biol. 2014;59:4525-4548.

48. McMillan K, Bostani M, Cagnon CH, Yu L, Leng S, McCollough $\mathrm{CH}$, et al. Estimating patient dose from CT exams that use automatic exposure control: Development and validation of methods to accurately estimate tube current values. Med Phys. 2017;44: 4262-4275.

49. Schlattl H, Zankl M, Becker J, Hoeschen C. Dose conversion coefficients for CT examinations of adults with automatic tube current modulation. Phys Med Biol. 2010;55:6243-6261.

50. Schlattl H, Zankl M, Becker J, Hoeschen C. Dose conversion coefficients for paediatric CT examinations with automatic tube current modulation. Phys Med Biol. 2012;57:6309-6326.

51. Hardy AJ, Angel E, Bostani M, Cagnon CH, McNitt-Gray M. Estimating fetal dose from tube current-modulated (TCM) and fixed tube current (FTC) abdominal/pelvis CT examinations. Med Phys. 2019;46:2729-2743.

52. Lee C, Kuzmin GA, Bae J, Yao J, Mosher E, Folio LR. Automatic mapping of CT scan locations on computational human phantoms for organ dose estimation. J Digit Imaging. 2019;32:175-182.

53. Choy G, Khalilzadeh O, Michalski M, Do S, Samir AE, Pianykh OS, et al. Current applications and future impact of machine learning in radiology. Radiology. 2018;288:318-328.

54. Lee C, Liu J, Griffin K, Folio LR, Summers RM. Adult patientspecific CT organ dose estimations using automated segmentations and Monte Carlo simulations. Biomed Phys Eng Express. 2020;6:045016.

55. Sharma S, Kapadia A, Fu W, Abadi E, Segars WP, Samei E. A realtime Monte Carlo tool for individualized dose estimations in clinical CT. Phys Med Biol. 2019;64:215020.

56. Chen W, Kolditz D, Beister M, Bohle R, Kalender WA. Fast on-site Monte Carlo tool for dose calculations in CT applications. Med Phys. 2012;39:2985-2996.

57. Ding A, Gu J, Trofimov AV, Xu XG. Monte Carlo calculation of imaging doses from diagnostic multidetector CT and kilovoltage cone-beam CT as part of prostate cancer treatment plans. Med Phys. 2010;37:6199-6204.

58. Ding GX, Coffey CW. Radiation dose from kilovoltage cone beam computed tomography in an image-guided radiotherapy procedure. Int J Radiat Oncol Biol Phys. 2009;73:610-617. 